\title{
Identification and clinical significance of somatic oncogenic mutations in epithelial ovarian cancer
}

\author{
Takafumi Watanabe ${ }^{1 *}$ CD, Hideaki Nanamiya ${ }^{2}$, Yuta Endo ${ }^{1}$, Manabu Kojima' ${ }^{1}$ Shinji Nomura', \\ Shigenori Furukawa', Shu Soeda' ${ }^{2}$, Hirosumi Tamura², Masae Ryufuku², Daisuke Tanaka², Takao Isogai², \\ Jun-ichi Imai ${ }^{2}$, Shinya Watanabe ${ }^{2}$ and Keiya Fujimori ${ }^{1}$
}

\begin{abstract}
Objective: Epithelial ovarian cancer (EOC) is a heterogeneous disease with diverse clinicopathological features and behaviors, and its heterogeneity may be concerned with the accumulation of multiple somatic oncogenic mutations. The major goals of this study are to systematically perform the comprehensive mutational profiling in EOC patients, and investigate the associations between somatic mutations and clinicopathological characteristics.
\end{abstract}

Methods: A total of 80 surgical specimens were obtained from EOC patients who had previously undergone primary debulking surgery, and genomic DNAs were extracted from fresh-frozen tissues. We investigated mutational status in hot spot regions of 50 cancer-related genes by targeted next-generation sequencing using an lon Ampliseq Cancer Hotspot Panel v2 Kit.

Results: Validated mutations were detected in 66 of the 80 tumors (82.5\%). The five most frequently mutated genes were TP53 (43.8\%), PIK3CA (27.5\%), KRAS (23.8\%), PTEN (10\%) and CTNNB1 (10\%). PTEN and CTNNB1 mutations were associated with younger age. PIK3CA1, KRAS and CTNNB1 mutations were observed in early-stage, whereas TP53 mutations were more common in advanced stage. Significant associations were observed between TP53 mutation and serous carcinoma, and between KRAS mutation and mucinous carcinoma. Both PIK3CA mutation and CTNNB1 mutation were also significantly associated with endometrioid and clear cell carcinoma. The patients with PIK3CA and KRAS mutations were significantly associated with favorable progression free survival (PFS). In particular, PIK3CA mutations had more significant associations with favorable PFS than PIK3CA wild-type in the endometrioid subtype $(P=0.012)$. Patients with mutations only in TP53 were significantly associated with worse PFS.

Conclusion: EOCs were heterogeneous at the genomic level and harbored somatic oncogenic mutations. Our molecular profiling may have the potential for becoming a novel stratification within histological subtypes of EOC. Further studies are needed to define molecular classification for improved clinical outcomes and treatment of EOC patients in future.

Keywords: Epithelial ovarian cancer, Somatic oncogenic mutations, Molecular profiling, Prognostic biomarker

*Correspondence: t-wata@fmu.ac.jp

1 Department of Obstetrics and Gynecology, Fukushima Medical University, Fukushima 960-1295, Japan

Full list of author information is available at the end of the article

\section{Introduction}

Epithelial ovarian cancer (EOC) is the seventh most common cancer in women and the eighth most common cause of death from cancer in women worldwide $[1,2]$. Recently, the incidence and mortality rates of patients original author(s) and the source, provide a link to the Creative Commons licence, and indicate if changes were made. The images or other third party material in this article are included in the article's Creative Commons licence, unless indicated otherwise in a credit line to the material. If material is not included in the article's Creative Commons licence and your intended use is not permitted by statutory regulation or exceeds the permitted use, you will need to obtain permission directly from the copyright holder. To view a copy of this licence, visit http://creativecommons.org/licenses/by/4.0/. The Creative Commons Public Domain Dedication waiver (http://creativeco mmons.org/publicdomain/zero/1.0/) applies to the data made available in this article, unless otherwise stated in a credit line to the data. 
with EOC have significantly increased. Although many surgical techniques and combination of chemotherapies have been widely promoted in clinical practice, the 5 -year survival rate of EOC patients is $47 \%$ [3]. Therefore, a better understanding of potential molecular mechanisms provides opportunities for early diagnosis and optimal management of EOC.

EOC is a heterogeneous disease comprised of multiple subtypes which exhibit diverse clinicopathologic features and behaviors. Recently, a dualistic model has been proposed to divide EOC into two broad categories called type I and type II [4]. Type I cancers include low-grade serous cancer, endometrioid cancer, mucinous cancer, and clear cell cancer, which harbor somatic mutations such as BRAF, KRAS and PTEN, often with microsatellite instability (MSI). They are characterized by an indolent behavior diagnosed in early-stage, and arise in a stepwise process from borderline neoplasms [5-7]. Type II cancers are clinically aggressive and comprise high-grade serous carcinoma (HG-SC), carcinosarcomas, and undifferentiated carcinomas, which are associated with mutations in TP53 and BRCA1/2 $[4,5]$. Although the classification of type I and II tumors better reflects the molecular diversity of EOC, the molecular biological heterogeneity of type I and II tumors has yet to be revealed.

Elucidating the relationship between genomic alterations and pathological factors can improve the clinical management and therefore survival rate of EOC patients, since it is now apparent that histological subtypes based primarily on morphology and immunohistochemistry tests are important for assessment in EOC. Several studies have revealed the genetic heterogeneity of each histological subtype such as serous carcinoma (SC), endometrioid carcinoma (EC), clear cell carcinoma (CCC) and mucinous carcinoma (MC) [8-11]. However, there have been few reports focusing on a systematic mutational landscape including all histological subtypes. In the present study, we performed comprehensive mutational profiling of EOC tumors using a cancer panel, and investigated the clinical significance of somatic oncogenic mutations.

\section{Materials and methods \\ Clinical samples}

A collection of specimens was obtained from EOC patients who had undergone primary debulking surgery at Fukushima Medical University Hospital between August 2013 and December 2017. Histopathological, clinical and treatment data were obtained from the patients' clinical, operative and pathological records. Taxane-platinum combination chemotherapy was given to all patients who were candidates for adjuvant treatment. The current study was a retrospective study, using genomic analyses, and was approved by the ethics committee of Fukushima Medical University (No. 1953). Written informed consent was obtained from all patients.

\section{Next-Generation Sequencing (NGS) for hotspot regions in $\mathbf{5 0}$ cancer-related genes}

Genomic DNAs were extracted from fresh-frozen tissue samples using ISOGEN reagent (Nippongene, Tokyo, Japan), according to the manufacturer's instructions. The quality and quantity of each DNA sample were assessed using NanoDrop One (ThermoFisher Scientific, Waltham, MA, USA).

The NGS for genomic DNAs from each sample was performed using the Ion Ampliseq Cancer Hotspot Panel v2, which covers approximately 2800 mutational hotspot regions from 50 cancer-related genes [12, 13]. In brief, $10 \mathrm{ng}$ of genomic DNAs extracted from 80 frozen tumor samples were used to construct barcoded DNA libraries utilizing an Ion Ampliseq Library Kit 2.0 (Thermo Fisher Scientific). The obtained libraries were optimized using an Ion Library Equalizer Kit (Thermo Fisher Scientific), and then sequenced using an Ion Personal Genome Machine or Ion S5XL platform (Thermo Fisher Scientific). The sequencing reads were aligned to the reference genome build hg19, GRCh37, and converted into BAM files using Ion Torrent Suite software (Thermo Fisher Scientific). Sequence variants were then called using Ion Reporter 5.0 (Thermo Fisher Scientific), according to the manufacturer's instructions. The mean read depth of coverage in DNA sequencing was over 1500 -fold.

\section{Statistical analysis}

The associations between somatic mutations and stage or histology were evaluated using the chi-squared test. Somatic mutation frequency in each of the four histological subtypes, SC, EC, CCC, and MC was analyzed using the Kruskal-Wallis test. Progression free survival (PFS) and overall survival (OS) were evaluated as clinical outcomes. The Kaplan-Meier analysis with log-rank test was used to compare survival distributions. In all analyses, statistical significance was defined as $P<0.05$. Statistical analysis was conducted using SPSS software version 25 (SPSS, Inc., Chicago, IL, USA).

\section{Results}

\section{Patients characteristics}

A total of 80 EOC patients who had undergone primary debulking surgery were enrolled in this study, and their clinicopathological characteristics are shown in Table 1 . The median age at diagnosis was 60 years (range, $36-89$ years), and 72 (90\%) patients with high risk of recurrence underwent taxane-platinum chemotherapy postoperatively. Among the 80 tumors, there were 32 
Table 1 Clinicopathological characteristics and mutational status of patients with epithelial ovarian cancer

\begin{tabular}{|c|c|c|}
\hline Characteristic & No. of cases & (\%) \\
\hline Median age, years (range) & $60(36-89)$ & \\
\hline \multicolumn{3}{|l|}{ Age } \\
\hline$<60$ & 39 & $(48.8)$ \\
\hline$\geq 60$ & 41 & $(51.3)$ \\
\hline \multicolumn{3}{|l|}{ Stage } \\
\hline । & 22 & $(27.5)$ \\
\hline$\|$ & 12 & $(15.0)$ \\
\hline III & 38 & $(47.5)$ \\
\hline IV & 7 & $(10.0)$ \\
\hline \multicolumn{3}{|l|}{ Histology } \\
\hline SC & 32 & $(40.0)$ \\
\hline $\mathrm{CCC}$ & 21 & $(26.3)$ \\
\hline EC & 20 & $(25.0)$ \\
\hline$M C$ & 7 & $(8.8)$ \\
\hline \multicolumn{3}{|l|}{ Mutational status } \\
\hline None & 14 & $(17.5)$ \\
\hline One & 36 & $(45.0)$ \\
\hline Two or more & 30 & $(37.5)$ \\
\hline Median PFS (months) (95\% Cl) & $39.2(32.5-45.9)$ & \\
\hline Median OS (months) $(96 \% \mathrm{CI})$ & $55.1(49.6-60.4)$ & \\
\hline 3-year PFS & 36 & $(45.0)$ \\
\hline 3-year OS & 59 & $(73.8)$ \\
\hline
\end{tabular}

SC Serous carcinoma, EM Endometrioid carcinoma, CCC Clear cell carcinoma, MC Mucinous carcinoma

(40.0\%) SC, 21 (26.3\%) CCC, 20 (25.0\%) EC, and seven (8.8\%) MC (Table 1). All SC tumors in this study were HG-SC tumors. After a median follow-up of 41 months (range, 1-71 months), 56 (70\%) patients were alive without clinical evidence of tumor. Recurrence was detected during the follow-up period in 43 (53.8\%) patients: 17 (21.3\%) patients were alive with disease; 24 (30\%) patients died due to tumor progression.

\section{Mutation frequencies in EOC}

We employed targeted NGS technology to explore somatic mutations occurring in EOC using 80 freshfrozen tumors. A summary of the relationships between somatic mutations and histological characteristics is described in Fig. 1. Validated mutations were found in 66 of the 80 tumors $(82.5 \%)$, and 30 of the 80 tumors (37.5\%) harbored concurrent mutations in two or more genes (Table 1). Mutations were found in 20 among the 50 tumor-related genes in EOC tumors. Mutations were most frequently detected in TP53 (43.8\%), PIK3CA (27.5\%) and KRAS (23.8\%) (Fig. 1). PTEN (10\%), CTNNB1 (10\%), FBFR2 (5\%) and FBXW7 (5\%) mutations were relatively frequent (Fig. 1 and Additional file 1). RB1 (2.5\%), APC, AKT1, ATM, ERBB4, SMO, STK11, EGFR1, GNAQ,
FLT3, CSF1R, EZH2 and VHL (1.3\%) were minor mutations (Fig. 1 and Additional file 1). A total of 114 (mean, 1.43) mutations were detected; 98 (86\%) missense mutations, 9 (7.9\%) nonsense mutations, 6 (5.3\%) frameshift indels and one $(0.8 \%)$ non-frameshift indels (Fig. 1 and Additional file 1).

The mean (SD) of mutation frequency was 1.43 (1.26) in all EOC patients, $0.94(0.61)$ in SC, 1.14 (0.83) in CCC, $1.71(0.45)$ in $\mathrm{MC}$ and 2.4 (1.88) in EC. The association between mutation frequency and each histological subtype was investigated by the Kruskal-Wallis test. Significant difference was identified in mutation frequency between SC vs MC $(P=0.017), \operatorname{SC}$ vs EC $(P=0.002)$ and CCC vs EC $(P=0.011)$ (Fig. 2).

\section{Associations of mutation status of TP53, PIK3CA, KRAS, PTEN or CTNNB with clinicopathological characteristics}

A summary of the associations between each of the five most frequently mutated genes (TP53, PIK3CA, KRAS, $P T E N$ and CTNNB1) and clinicopathological characteristics of the EOC patients is described in Table 2. Patients with PTEN and CTNNB1 mutations had a significantly younger age $(<60)$ than those without these mutations ( $P=0.02$ and $P=0.021$, respectively). CTNNB1, PIK3CA and $K R A S$ mutations were significantly detected in earlystage EOC $(P=0.001, P=0.004$ and $P=0.009$, respectively), whereas TP53 mutations were significantly more common in advanced-stage EOC $(P=0.026)$. Significant associations between each of the five mutated genes (TP53, PIK3CA, KRAS, PTEN and CTNNB1) and histological subtypes were found as shown in Table 2.

\section{Prognostic role of clinicopathological factors and genomic mutations in EOC}

The association between clinicopathological factors and survival is summarized in Table 3. The 3-year PFS and OS of the 80 patients were 50.0 and $75.0 \%$, respectively. Late-stage, histological subtype, PIK3CA wild-type (WT) (Fig. 3a) and KRAS WT (Fig. 3c) were significantly associated with worse PFS $(P<0.001, P<0.001, P=0.047$ and $P=0.043$, respectively), while age, TP53, PTEN and CTNNB1 were not. In particular, PIK3CA mutations had more significant associations with favorable PFS than PIK3CA WT in the EC subtype $(P=0.012)$ (Fig. $3 \mathrm{~b})$. Although TP53 mutations did not influence the EOC survival, in the subgroup analysis, patients with mutations only in TP53 were significantly associated with worse PFS compared to those with TP53 WT $(P=0.008)$ or those with TP53 mutation with other mutations $(P=0.006)$ (Fig. 3d) Regarding OS, as shown in Table 3, a significant association was only found with stage $(P<0.001)$, and age, histology, and mutations of TP53, PIK3CA, KRAS, PTEN and CTNNB1 had no significant associations. 


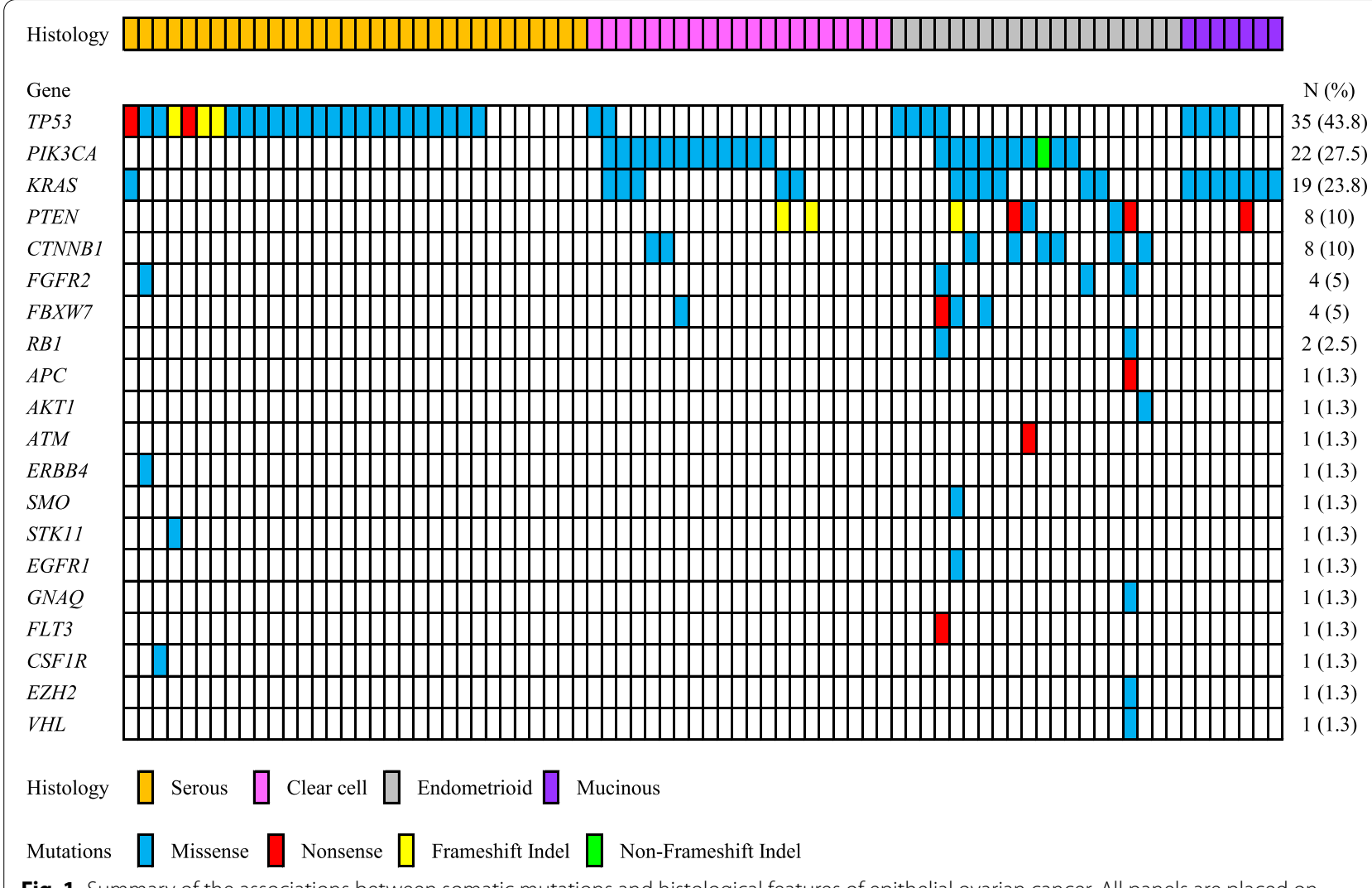

Fig. 1 Summary of the associations between somatic mutations and histological features of epithelial ovarian cancer. All panels are placed on vertical tracks representing 80 individuals

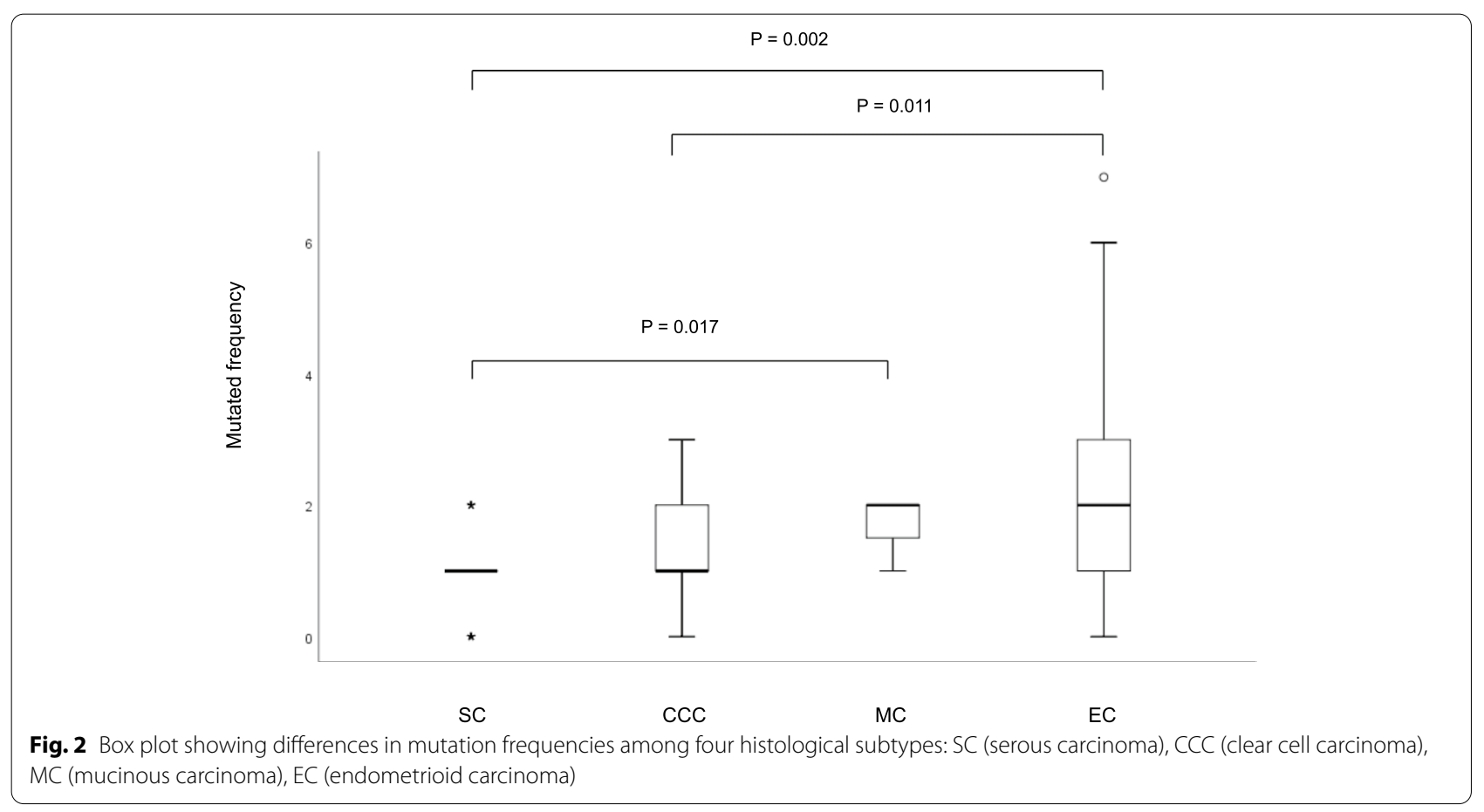


Table 2 Frequency of TP53, PIK3CA, KRAS, PTEN and CTNNB mutations according to demographic and clinicopathological characteristics

\begin{tabular}{|c|c|c|c|c|c|c|c|c|c|c|c|}
\hline & \multicolumn{2}{|l|}{ Age } & \multirow[t]{2}{*}{$P$ value } & \multicolumn{2}{|c|}{ Stage } & \multirow[t]{2}{*}{$P$ value } & \multicolumn{4}{|c|}{ Histology } & \multirow[t]{2}{*}{$P$ value } \\
\hline & $<60$ & $\geq 60$ & & I/II & III/IV & & Serous & Clear cell & Endometrioid & Mucinous & \\
\hline \multicolumn{12}{|l|}{ TP53 } \\
\hline WT & 25 & 20 & & 24 & 21 & & 7 & 19 & 16 & 3 & \\
\hline MT & 14 & 21 & 0.17 & 10 & 25 & 0.026 & 25 & 2 & 4 & 4 & $<0.001$ \\
\hline \multicolumn{12}{|l|}{ PIK3CA } \\
\hline WT & 26 & 32 & & 19 & 39 & & 32 & 9 & 10 & 7 & \\
\hline MT & 13 & 9 & 0.25 & 15 & 7 & 0.004 & 0 & 12 & 10 & 0 & $<0.001$ \\
\hline \multicolumn{12}{|l|}{ KRAS } \\
\hline WT & 30 & 31 & & 21 & 40 & & 31 & 16 & 14 & 0 & \\
\hline MT & 9 & 10 & 0.89 & 13 & 6 & 0.009 & 1 & 5 & 6 & 7 & $<0.001$ \\
\hline \multicolumn{12}{|l|}{ PTEN } \\
\hline WT & 31 & 41 & & 31 & 41 & & 32 & 19 & 15 & 6 & \\
\hline MT & 8 & 0 & 0.002 & 4 & 4 & 0.71 & 0 & 2 & 5 & 1 & 0.033 \\
\hline \multicolumn{12}{|c|}{ CTNNB1 } \\
\hline WT & 32 & 40 & & 27 & 45 & & 32 & 19 & 14 & 7 & \\
\hline MT & 7 & 1 & 0.021 & 8 & 0 & 0.001 & 0 & 2 & 6 & 0 & 0.004 \\
\hline
\end{tabular}

WT Wild-type, MT Mutant

\section{Discussion}

Somatic oncogenic mutations are actionable mutations in EOC patients. Validated mutations have been reported in $71-89 \%$ of EOC tumors using a cancer panel $[14,15]$, and the current study had a similar rate $(82.5 \%)$. Sixty-six of the 80 EOC patients had one or more somatic mutations in either one or multiple genes (Table 1). We examined the association between somatic mutation frequencies and four histological subtypes of EOC. EC was found to have the highest frequency of mutations in the present study (Fig. 2). As a consequence of mismatch repair (MMR) deficiency due to mutations or methylation in MMR protein-encoding genes, tumors with MSI accumulate high numbers of mutations. EC tumors may have had higher MSI than other histological tumors.

We here examined the relationships between the five most frequently mutated genes and clinicopathological features or prognosis in EOC patients. p53 proteins involve in cell-cycle checkpoints, senescence, DNA repair, apoptosis and cellular stress responses. The TP53 gene is one of the most commonly inactivated tumor suppressors in human cancer [16]. TP53 was also identified to be mutated at the highest frequency in the current study (Fig. 1). In particular, TP53 mutations were most frequently observed in SC $(78 \%, 25 / 32)$ among the four histological subtypes (Table 2). According to The Cancer Genome Atlas (TCGA) Research Network, TP53 mutations occur in 96\% of HG-SC tumors [8]. The mutation spectrum completely separates HG-SC from other histological subtypes of EOC; in HG-SC, somatic mutations other than TP53 mutations are rare. While it is well accepted that TP53 mutation is an essential event in the genesis of HG-SC, we showed that mutant p53 was often seen in MC $(57.1 \%, 4 / 7)$, which is consistent with previous studies $[9,17]$. On the other hands, the frequencies of TP53 mutation in CCC 9.5\% (2/21) and EC 20\% $(4 / 20)$ were lower. Cybulska P and Kim SI reported that the frequency of TP53 mutations was 17\% (6/36) and 13\% $(2 / 15)$ in EC and CCC, respectively [10, 11]. The association of TP53 mutations with late-stage EOC was statistically significant in the current study. The reason is that most of HG-SC with TP53 mutations are diagnosed in an advanced stage, and the other histological subtypes (EC, CCC, and $\mathrm{MC}$ ) that more commonly present at an early stage have a much lower incidence of TP53 mutations. TP53 mutations had marginally significant associations with poor PFS in the present study. Furthermore, patients with mutations only in TP53 had a significantly worse PFS compared to those with no TP53 mutations and those with TP53 mutations with other mutations (Fig. 3d). Although TP53 mutation was often seen in MC $(57.1 \%, 4 / 7)$, all patients with MC harbored KRAS mutation. Table 3 shows that patients with HG-SC had significantly worse PFS than patients with other histological subtypes. Since patients with mutation only in TP53 had a high rate of HG-SC (Fig. 1), they are predicted to have a poor prognosis.

PIK3CA encodes a lipid kinase involved in multiple signaling pathways that influence cellular functions such as growth, death, and proliferation. PIK $3 C A$ 
Table 3 The relationship of patients' clinicopathological characteristics and somatic mutations with progression free survival and overall survival

\begin{tabular}{|c|c|c|c|c|c|}
\hline & $\mathrm{N}$ & 3-Year RFS (\%) & $P$-value & 3-Year OS (\%) & $P$-value \\
\hline Age (years) & & & 0.17 & & 0.31 \\
\hline$<60$ & 38 & 55.3 & & 78.9 & \\
\hline$\geq 60$ & 42 & 45.2 & & 71.4 & \\
\hline Stage & & & $<0.001$ & & $<0.001$ \\
\hline |/I| & 34 & 79.4 & & 93.9 & \\
\hline III/IV & 46 & 28.3 & & 63.0 & \\
\hline Histology & & & $<0.001$ & & 0.072 \\
\hline SC & 32 & 17.9 & & 67.9 & \\
\hline $\mathrm{CCC}$ & 21 & 47.6 & & 61.9 & \\
\hline EC & 20 & 80 & & 85.0 & \\
\hline$M C$ & 7 & 87.5 & & 100 & \\
\hline TP53 & & & 0.088 & & 0.74 \\
\hline WT & 45 & 57.8 & & 75.6 & \\
\hline MT & 35 & 31.4 & & 74.3 & \\
\hline PIK3CA & & & 0.047 & & 0.74 \\
\hline WT & 58 & 43.1 & & 75.9 & \\
\hline MT & 22 & 68.2 & & 72.7 & \\
\hline KRAS & & & 0.043 & & 0.11 \\
\hline WT & 61 & 44.2 & & 72.1 & \\
\hline MT & 19 & 68.4 & & 84.2 & \\
\hline PTEN & & & 0.58 & & 0.84 \\
\hline WT & 72 & 48.6 & & 75.0 & \\
\hline MT & 8 & 62.5 & & 75.0 & \\
\hline CTNNB1 & & & 0.59 & & 0.89 \\
\hline WT & 72 & 48.6 & & 75.0 & \\
\hline MT & 8 & 62.5 & & 75.0 & \\
\hline
\end{tabular}

SC Serous carcinoma, EM Endometrioid carcinoma, CCC Clear cell carcinoma, MC Mucinous carcinoma, WT Wild-type, MT Mutant

mutations occur in about $13 \%$ of solid tumors [18], and in our study, they occurred in $27.5 \%(22 / 80)$ of the EOC, which was similar to a recent study [19]. As for histological subtypes of EOC, PIK3CA mutation is rare in HG-SC and MC, although may be seen in up to $40 \%$ of CCC and EC [20], similar to our results (Table 2). PIK3CA mutations had significant associations with favorable PFS $(P=0.047)$ (Fig. 3a). In particular, we found PIK3CA mutations had more significant associations with favorable PFS than PIK3CA wild-type in the in the EC subtype $(P=0.012)$ (Fig. $3 \mathrm{~b})$. This significant prognostic value of PIK3CA mutation status in ECs is potentially interesting, and, to the best of our knowledge, has not been reported. In previous reports, PI3K pathway activation including the PIK3CA mutation was associated with favorable prognosis in CCC [21, 22]. Although PIK3CA mutation status was not a significant prognostic factor in CCC in our study, further studies with larger study populations are necessary to evaluate whether inactivation of PIK3CA is a useful biomarker.

The KRAS gene belongs to the Ras family of oncogenes and encodes the K-Ras protein, which is a part of the tyrosine kinase signaling RAS/MAPK pathway. KRAS mutations are the most frequent mutations in human cancer, and are found in up to $25 \%$ of all human cancers [23]. In the results of our study, KRAS mutations were detected in $23.8 \%(19 / 80)$ and correlated with early-stage, $\mathrm{MC}$ and better PFS. In the previous studies, KRAS mutations were reported to be related to type I histology types, especially their high frequency was reported in MCs and low-grade SCs $[20,24,25]$. In the present study, all of the MC tumors were observed to have KRAS mutations. As for prognosis, KRAS mutations have been proven to present in type I tumors characterized by early-stage and indolent behavior, and hence, generally associated with a more favorable survival [25].

PTEN inactivation results in abnormal cell growth and apoptosis escape. PTEN is known to be one of the most frequently mutated tumor suppressor genes in human cancer. Among several studies in EOC, the frequencies of PTEN mutations have been reported to be $5-21 \%$ [26-28], and said frequency in the present study was $10 \%$ (8/80). Regarding histological subtypes, PTEN mutations have been identified predominantly in type I, but not in type II [29]. In the current study, somatic mutations in $P T E N$ were most frequently observed in EC (5/20, 25\%). Dinulescu et al. reported that the combination of PTEN and KRAS mutations in the ovary induced invasive and widely metastatic EC [30]. They also suggest that aberrant activation of the PI3K/Akt/mTOR pathway may lead to the tumorigenesis and development of EC.

The $\beta$-catenin (CTNNB1) inactivation results in the activation of the Wnt signaling pathway, which plays an important role in the regulation of cell proliferation, differentiation tissue homeostasis, migration, embryonic development, cell fate determination, and self-renewal in stem cells [31]. Aberrant activation of Wnt signaling has been specifically shown to be associated with numerous malignancies [32]. Somatic mutations of CTNNB1 were frequently observed in EC $(30 \%, 6 / 20)$ and CCC $(9.5 \%$, $2 / 21$ ) in the present study (Table 2). CTNNB1 mutations are the most common genetic alterations identified in EC [11]. Not as much as EC tumors, CTNNB1 mutations are present in approximately $10 \%$ of CCC tumors [33]. On the other hand, no CTNNB1 mutations were observed in $\mathrm{SC}$ or $\mathrm{MC}$ in our study, which is similar to previous reports $[8,9]$, and this tendency was also observed in PIK3CA or PTEN mutations in our study. Lac et al. described hotspot mutations in KRAS, ERBB2, PIK3CA and CTNNB1, as well as heterogeneous PTEN loss and ARID1A loss in endometriosis [34]. Since it is thought 


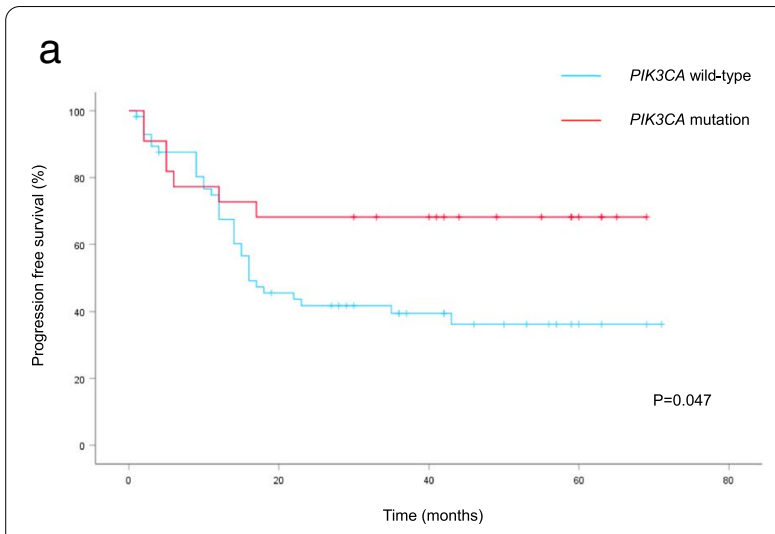

C

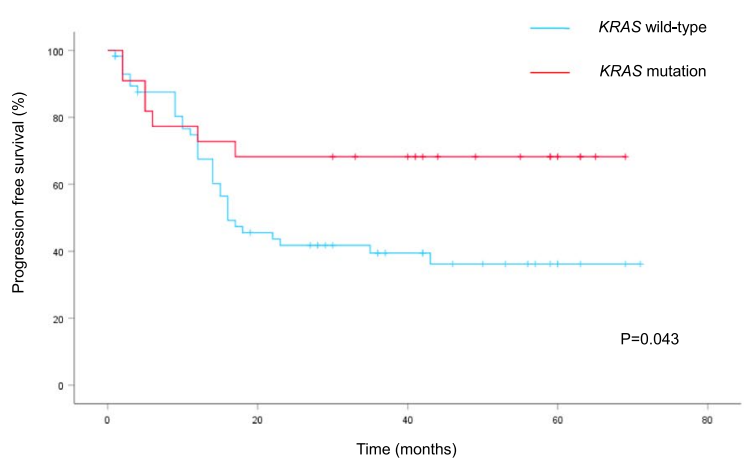

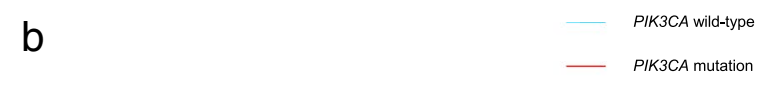

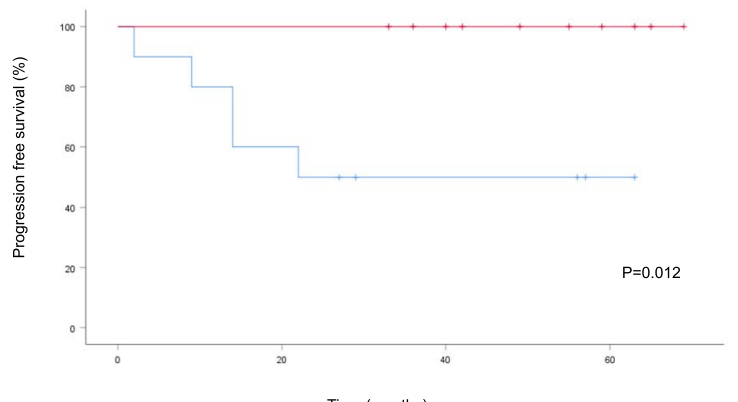

d

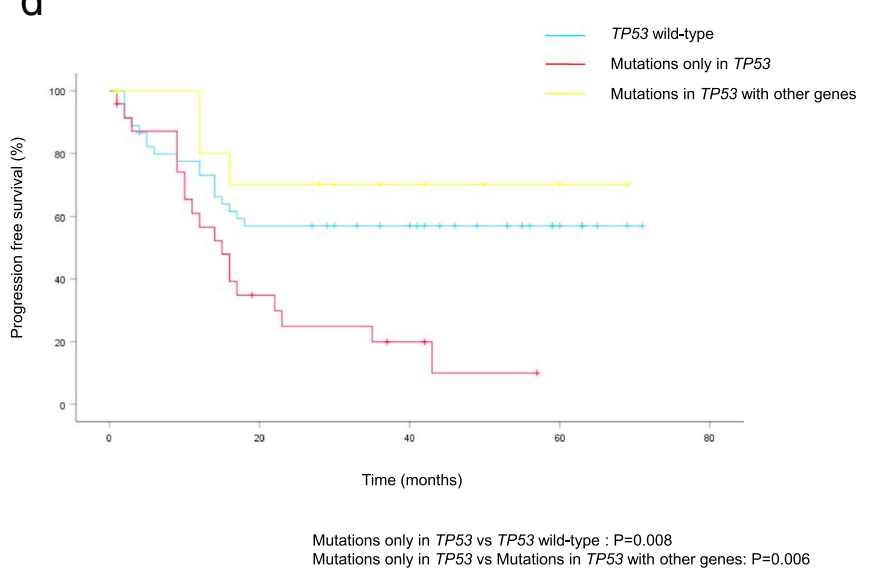

Fig. 3 The Kaplan-Meier curves of progression free survival in patients with epithelial ovarian cancer. aPIK3CA mutations and wild-type. $\mathbf{b} P I K 3 C A$ mutations and wild-type in the patients with endometrioid carcinoma. cKRAS mutations and wild-type. d Mutations only in TP53, TP53 wild-type, and mutations in TP53 with other genes

that endometriosis-associated cancers include CCC and $\mathrm{EC}$, gene mutations in endometriosis and these lesions may be similar $[35,36]$. The associations of CTNNB1 mutations with younger age and early-stage were significant in the present study. The reason is that the patients with CCC and EC, which are included in type I, were younger than those with HG-SC, which is included in type II. Interestingly, this tendency was also observed in uterine endometrial cancer [37].

The main limitations of our study were its retrospective design, the small sample size, histological heterogeneity, and short follow-up period. Therefore, a prospective study using a larger sample size with long-term follow-up would likely provide more reliable results. Another limitation of the current study was that our mutation analysis may have lacked any relevant targetable mutations due to a small hotspot panel. The use of genome-wide sequencing such as whole exome or genome sequencing would make it possible to detect more actionable gene mutations. Finally, we did not evaluate homologous recombination deficiency (HRD), BRCA1/2 mutations and deficient MMR (dMMR). TCGA suggests that approximately $50 \%$ of HG-SC indicate HRD due to genetic and epigenetic alterations of HR pathway genes [8]. BRCA1/2 mutations are the most common genetic cause of HRD. Approximately $15 \%$ of EOC cases are associated with germline $B R C A 1 / 2$ pathogenic variants, and additional $5 \%$ show somatic $B R C A 1 / 2$ mutations [38, $39]$. Although a total of approximately $20 \%$ of $B R C A 1 / 2$ mutations should have been detected in the EOC tumors, the gene panel we used did not carry BRCA1/2 genes. dMMR is estimated to occur in approximately $10 \%$ of EOC [40]. The reported frequency of MSI in EC ranges from 10 to $19 \%$ [11, 41, 42]. Rambau PF et al. have reported that $\mathrm{DMMR}$ was detected in $13.8 \%$ of $\mathrm{EC}$ and $2.4 \%$ of CCC, but not MC or SC using immunohistochemistry for MMR proteins [43]. Although we did not perform MSI assay or immunohistochemistry for MMR proteins in the present study, the result that EC had the highest frequency of mutations implied that EC tumors have the highest frequency of dMMR among histological subtypes of EOC [44]. Since HRD, including BRCA1/2 mutations, and dMMR have recently emerged as predictive biomarkers for the choice of poly (ADP-ribose) 
polymerase inhibitor and immune checkpoint inhibitor, respectively, these analyses should have been performed in our study.

\section{Conclusion}

We demonstrated that our comprehensive gene mutation profiles using targeted next generation sequencing panel in EOC are feasible. Although the clinical significance of somatic oncogenic mutations in EOC patients was similar to that detected in previous studies, we revealed the associations between PIK3CA mutation and better PFS in EC and between mutations only in TP53 and worse PFS. This work will be useful to understand and evaluate the molecular features of EOC patients, and may help to establish future novel treatment strategies that improve outcome in EOC patients.

\section{Supplementary Information}

The online version contains supplementary material available at https://doi. org/10.1186/s13048-021-00876-z.

Additional file 1. Summary of somatic oncogenic mutation status in 80 patients with epithelial ovarian cancer.

\section{Acknowledgments}

Not applicable.

\begin{abstract}
Authors' contributions
Takafumi Watanabe: Conceptualization, Methodology, Investigation, Writing an Original Draft. Hideaki Nanamiya, Hirosumi Tamura, Masae Ryufuku, Daisuke Tanaka, Takao Isogai and Jun-ichi Imai: Data curation, Formal analysis. Yuta Endo, Manabu Kojima, Shinji Nomura, Shigenori Furukawa and Shu Soeda: Resources, Investigation. Shinya Watanabe: Project administration, Funding acquisition. Keiya Fujimori: Supervised writing of the paper. All authors read and approved the final manuscript.
\end{abstract}

\section{Funding}

The present study was supported by grants for Translational Research Programs from Fukushima Prefecture.

\section{Availability of data and materials}

The datasets used and analyzed during the present study are available from the corresponding author on reasonable request.

\section{Declarations}

\section{Ethics approval and consent to participate}

The study protocol was approved by the Ethics Committee of Fukushima Medical University School of Medicine (No. 1953), and informed consent was obtained from all patients. All participants signed informed consent forms.

\section{Consent for publication}

Not applicable.

\section{Competing interests}

The authors declare that they have no competing interests.

\section{Author details}

${ }^{1}$ Department of Obstetrics and Gynecology, Fukushima Medical University, Fukushima 960-1295, Japan. ${ }^{2}$ Translational Research Center, Fukushima Medical University, Fukushima 960-1295, Japan.
Received: 12 April 2021 Accepted: 6 September 2021

Published online: 06 October 2021

\section{References}

1. Webb PM, Jordan SJ. Epidemiology of epithelial ovarian cancer. Best Pract Res Clin Obstet Gynaecol. 2017;41:3-14.

2. Gaona-Luviano P, Medina-Gaona LA, Magaña-Pérez K. Epidemiology of ovarian cancer. Chin Clin Oncol. 2020;9:47.

3. Smith RA, Andrews KS, Brooks D, Fedewa SA, Manassaram-Baptiste D, Saslow D, et al. Cancer screening in the United States, 2018: a review of current American Cancer Society guidelines and current issues in cancer screening. CA Cancer J Clin. 2018;68:297-316.

4. Kurman RJ, Shih I-M. Molecular pathogenesis and extraovarian origin of epithelial ovarian cancer: shifting the paradigm. Hum Pathol. 2011;42:918-31.

5. Banerjee S, Kaye SB. New strategies in the treatment of ovarian cancer: current clinical perspectives and future potential. Clin Cancer Res. 2013;19:961-8

6. Kossaï M, Leary A, Scoazec JY, Genestie C. Ovarian cancer: a heterogeneous disease. Pathobiology. 2018;85:41-9.

7. Chen S, Li Y, Qian L, Deng S, Liu L, Xiao W, et al. A review of the clinical characteristics and novel molecular subtypes of endometrioid ovarian cancer. Front Oncol. 2021;11:668151.

8. Cancer Genome Atlas Research Network. Integrated genomic analyses of ovarian carcinoma. Nature. 2011;474:609-15.

9. Ryland GL, Hunter SM, Doyle MA, Caramia F, Li J, Rowley SM, et al. Mutational landscape of mucinous ovarian carcinoma and its neoplastic precursors. Genome Med. 2015;7:87.

10. Kim SI, Lee JW, Lee M, Kim HS, Chung HH, Kim JW, et al. Genomic landscape of ovarian clear cell carcinoma via whole exome sequencing. Gynecol Oncol. 2018;148:375-82.

11. Cybulska P, Paula ADC, Tseng J, Leitao MM Jr, Bashashati A, Huntsman DG, et al. Molecular profiling and molecular classification of endometrioid ovarian carcinomas. Gynecol Oncol. 2019;154:516-23.

12. Ab Mutalib NS, Syafruddin SE, Md Zain RR, Mohd Dali AZ, Mohd Yunos $\mathrm{Rl}$, Saidin S, et al. Molecular characterization of serous ovarian carcinoma using a multigene next generation sequencing cancer panel approach. BMC Res Notes. 2014;7:805.

13. Watanabe T, Nanamiya H, Kojima M, Nomura S, Furukawa S, Soeda S, et al. Clinical implication of oncogenic somatic mutations in early-stage cervical cancer with radical hysterectomy. Sci Rep. 2020;10:18734.

14. Boland GM, Piha-Paul SA, Subbiah V, Routbort M, Herbrich SM, Baggerly $\mathrm{K}$, et al. Clinical next generation sequencing to identify actionable aberrations in a phase I program. Oncotarget. 2015;6:20099-110.

15. Wang M, Fan W, Ye M, Tian C, Zhao L, Wang J, et al. Molecular profiles and tumor mutational burden analysis in Chinese patients with gynecologic cancers. Sci Rep. 2018;8:8990.

16. Blandino G, Di Agostino S. New therapeutic strategies to treat human cancers expressing mutant p53 proteins. J Exp Clin Cancer Res. 2018;37:30

17. Mackenzie R, Kommoss S, Winterhoff BJ, Kipp BR, Garcia JJ, Voss J, et al. Targeted deep sequencing of mucinous ovarian tumors reveals multiple overlapping RAS-pathway activating mutations in borderline and cancerous neoplasms. BMC Cancer. 2015;15:415.

18. Alqahtani A, Ayesh HSK, Halawani H. PIK3CA gene mutations in solid malignancies: association with clinicopathological parameters and prognosis. Cancers (Basel). 2019;12:93.

19. Ogasawara A, Hihara T, Shintani D, Yabuno A, Ikeda Y, Tai K, et al. Evaluation of circulating tumor DNA in patients with ovarian cancer harboring somatic PIK3CA or KRAS mutations. Cancer Res Treat. 2020;52:1219-28.

20. Cheaib B, Auguste A, Leary A. The PI3K/Akt/mTOR pathway in ovarian cancer: therapeutic opportunities and challenges. Chin J Cancer. 2015;34:4-16.

21. Itamochi H, Oishi T, Oumi N, Takeuchi S, Yoshihara K, Mikami M, et al. Whole-genome sequencing revealed novel prognostic biomarkers and promising targets for therapy of ovarian clear cell carcinoma. Br J Cancer. 2017;117:717-24 
22. Rahman M, Nakayama K, Rahman MT, Nakayama N, Ishikawa M, Katagiri A, et al. Clinicopathologic and biological analysis of PIK3CA mutation in ovarian clear cell carcinoma. Hum Pathol. 2012;43:2197-206.

23. Kranenburg O. The KRAS oncogene: past, present, and future. Biochim Biophys Acta. 2005;1756:81-2.

24. Gemignani ML, Schlaerth AC, Bogomolniy F, Barakat RR, Lin O, Soslow $R$, et al. Role of KRAS and BRAF gene mutations in mucinous ovarian carcinoma. Gynecol Oncol. 2003;90:378-81.

25. Nodin B, Zendehrokh N, Sundström M, Jirström K. Clinicopathological correlates and prognostic significance of KRAS mutation status in a pooled prospective cohort of epithelial ovarian cancer. Diagn Pathol. 2013;8:106

26. Obata K, Morland SJ, Watson RH, Hitchcock A, Chenevix-Trench G, Thomas EJ, et al. Frequent PTEN/MMAC mutations in endometrioid but not serous or mucinous epithelial ovarian tumors. Cancer Res. 1998:58:2095-7.

27. Kurose K, Zhou XP, Araki T, Cannistra SA, Maher ER, Eng C. Frequent loss of PTEN expression is linked to elevated phosphorylated Akt levels, but not associated with p27 and cyclin D1 expression, in primary epithelial ovarian carcinomas. Am J Pathol. 2001:158:2097-106.

28. Kolasa IK, Rembiszewska A, Janiec-Jankowska A, Dansonka-Mieszkowska A, Lewandowska AM, Konopka B, et al. PTEN mutation, expression and $\mathrm{LOH}$ at its locus in ovarian carcinomas. Relation to TP53, K-RAS and BRCA1 mutations. Gynecol Oncol. 2006;103:692-7.

29. Nero C, Ciccarone F, Pietragalla A, Scambia G. PTEN and gynecological cancers. Cancers (Basel). 2019;11:1458.

30. Dinulescu DM, Ince TA, Quade BJ, Shafer SA, Crowley D, Jacks T. Role of K-ras and Pten in the development of mouse models of endometriosis and endometrioid ovarian cancer. Nat Med. 2005;11:63-70.

31. Anastas JN, Moon RT. WNT signalling pathways as therapeutic targets in cancer. Nat Rev Cancer. 2013;13:11-26.

32. Klaus A, Birchmeier W. Wnt signaling and its impact on development and cancer. Nat Rev Cancer. 2008;8:387-98.

33. Maru Y, Tanaka N, Ohira M, Itami M, Hippo Y, Nagase H. Identification of novel mutations in Japanese ovarian clear cell carcinoma patients using optimized targeted NGS for clinical diagnosis. Gynecol Oncol. 2017;144:377-83.

34. Lac V, Verhoef L, Aguirre-Hernandez R, Nazeran TM, Tessier-Cloutier B, Praetorius T, et al. latrogenic endometriosis harbors somatic cancer-driver mutations. Hum Reprod. 2019;34:69-78.
35. Anglesio MS, Yong PJ. Endometriosis-associated ovarian cancers. Clin Obstet Gynecol. 2017;60:711-27.

36. Hossain MM, Nakayama K, Shanta K, Razia S, Ishikawa M, Ishibashi T, et al. Establishment of a novel in vitro model of endometriosis with oncogenic KRAS and PIK3CA mutations for understanding the underlying biology and molecular pathogenesis. Cancers (Basel). 2021;13:3174

37. Watanabe T, Nanamiya H, Kojima M, Nomura S, Furukawa S, Soeda S, et al Clinical relevance of oncogenic driver mutations identified in endometrial carcinoma. Transl Oncol. 2021;14:101010.

38. Alsop K, Fereday S, Meldrum C, deFazio A, Emmanuel C, George J, et al. BRCA mutation frequency and patterns of treatment response in BRCA mutation-positive women with ovarian cancer: a report from the Australian Ovarian Cancer Study Group. J Clin Oncol. 2012;30:2654-63.

39. Stavropoulou AV, Fostira F, Pertesi M, Tsitlaidou M, Voutsinas GE, Triantafyllidou $\mathrm{O}$, et al. Prevalence of BRCA1 mutations in familial and sporadic greek ovarian cancer cases. PLoS One. 2013;8:e58182.

40. Murphy MA, Wentzensen N. Frequency of mismatch repair deficiency in ovarian cancer: a systematic review. This article is a US Government work and, as such, is in the public domain of the United States of America. Int J Cancer. 2011:129:1914-22.

41. Aysal A, Karnezis A, Medhi I, Grenert JP, Zaloudek CJ, Rabban JT. Ovarian endometrioid adenocarcinoma: incidence and clinical significance of the morphologic and immunohistochemical markers of mismatch repair protein defects and tumor microsatellite instability. Am J Surg Pathol. 2012;36:163-72.

42. Hollis RL, Thomson JP, Stanley B, Churchman M, Meynert AM, Rye T, et al. Molecular stratification of endometrioid ovarian carcinoma predicts clinical outcome. Nat Commun. 2020;11:4995.

43. Rambau PF, Duggan MA, Ghatage P, Warfa K, Steed H, Perrier R, et al. Significant frequency of MSH2/MSH6 abnormality in ovarian endometrioid carcinoma supports histotype-specific Lynch syndrome screening in ovarian carcinomas. Histopathology. 2016;69:288-97.

44. Fraune C, Rosebrock J, Simon R, Hube-Magg C, Makrypidi-Fraune G, Kluth $M$, et al. High homogeneity of MMR deficiency in ovarian cancer. Gynecol Oncol. 2020;156:669-75.

\section{Publisher's Note}

Springer Nature remains neutral with regard to jurisdictional claims in published maps and institutional affiliations.
Ready to submit your research? Choose BMC and benefit from:

- fast, convenient online submission

- thorough peer review by experienced researchers in your field

- rapid publication on acceptance

- support for research data, including large and complex data types

- gold Open Access which fosters wider collaboration and increased citations

- maximum visibility for your research: over $100 \mathrm{M}$ website views per year

At BMC, research is always in progress.

Learn more biomedcentral.com/submissions 